\title{
Chapter 43
}

\section{MODEL STUDY OF AN ISOLATED LIGHTHOUSE PLATFORM AT SEA (PRINCE SHOAL, QUEBEC)}

\author{
G. E. Jarlan
}

Ingineer, Hydraulics Laboratory

National. Research Council of Canada

\section{THEORETTCAL CONSTDERATIONS}

Beperiments on objects subject to wave forces have been made in the past, which led to formulae where the geometrical characteristics of the object are of primary importance. Other experiments, made on inmersed objects, showed that the accelerative forces caused by wave impact are of the same order of magnitude as the drag forces associated with orbital velocities. In the case of a small free bod completely immersed in a fluid and subject to a system of forces, other experiments showed that the fluid surrounding the body acquires a velocity while the flow fleld gains kinetic energy (Ref. 1). The acceleration of the object can be deduced from the pressure distribution around the body. For small obfects, the integrated form of the dynamical equation is:

$$
F=M \frac{\partial u}{\partial t}
$$

where $M=$ mass of fluid displaced by the object

$$
\frac{\partial U}{\partial t}=\text { acceleration normal to the object. }
$$

Since viscous friction is present, the notion of drag force expressed by the following relation must be used.

$$
F_{D}=C_{D} A \rho \frac{U^{2}}{2}
$$

where

$$
\begin{aligned}
\mathbf{A} & =\text { projected area of object } \\
\mathbf{c}_{\mathbf{D}} & =\text { drag coefficient } \\
\boldsymbol{P} & =\text { specific weight of water } \\
\mathbf{u} & =\text { horizontal component of velocity. }
\end{aligned}
$$

A combination of equations (1) and (2) yields the total force acting on the immersed free body, assuming the object has very small dimensions.

In the case of a cylindrical pile anchored to the bottom or 


\section{MODEL STUDY OF AN ISOLATED LIGHTHOUSE \\ PLATFORM AT SEA (PRINCE SHOAL, QUEBEC)}

protruding beyond the free surface and subject to wave fronts, Morison et al (Ref. 1) have shown that the tangential component of the force per unit length of eylinder can be expressed by:

$$
f_{x}=C_{D} \cdot \frac{P_{2}}{2 g} \cdot D \cdot v_{x}^{2}+C_{m} \cdot \frac{\rho}{g} \cdot \frac{D^{2}}{4} a_{x}
$$

where $C_{D}$ and $C_{m}$ are respectively the drag and virtual mass coefficients,

$$
\begin{gathered}
P=\text { specific weight of water } \\
D=\text { diameter of the pile } \\
\nabla_{x} \text { and } a_{x} \begin{array}{l}
\text { are the horizontal components of } \\
\text { velocity and acceleration }
\end{array} \\
C_{D} \text { and } C_{m} \begin{array}{l}
\text { appear to be functions of the Reynolds } \\
\text { number at the pile, hence they depend } \\
\text { also on } \nabla_{x}
\end{array}
\end{gathered}
$$

According to equation (3) the value of $f_{x}$ varies as the second power of D. However, this formula makes obseure the concept of energy dissipation by wave scattering. This phenomenon plays an important role in the process of energy dissipation since it is a function of the relative size of the obstacle with respect to the characteristics of the perturbation.

An interesting demonstration of this fact is given by Rayleigh (Ref. 2). A cylindrical obstacle is subject to the disturbance created by plane waves. Let $\varnothing$ and represent respectively the velocity potential of the incident and scattered waves; the plane waves can be represented by

$\phi=e^{i k a t} \cdot e^{i k r}$ (for normal incidence).

Dropping $e^{\text {ikat }}$ for sake of brevity, $\phi$ can hence be expressed as a series of Bessel functions of the form:

$$
\begin{aligned}
e^{i k r} & =J_{0}(k r)+2 i J(k r)+\ldots .+2 i^{x} J(k r) \\
\text { or } \quad \Phi & =1-\frac{k^{2}}{4} \rho^{2}+i k r
\end{aligned}
$$

where $r$ is the radius of the cylinder.

If it is assumed that the obstacle is composed of an isotropic 
material, the velocity potential, under normal incidence, of the scattered waves for a rigid and inmovable obstacle is:

$$
\psi=-\frac{2 \pi}{r^{2} / 2} \cdot \frac{\pi r^{2}}{\lambda^{2 / 2}} \cdot \cos \left(\frac{2 \pi r}{\lambda}+\frac{\pi}{4}\right)
$$

with $r=$ radius of the obstacle, $\lambda=$ wave length of the incident wave.

It is seen that the intensity of the scattered waves will be a function of the diameter of the obstacle relative to the wave-length of the perturbation. This explains why fog, which consists of spherical particles of condensed water vapour, lets sound waves pass through it, while light, the wave-length of which is small compared with the dimensions of the particles, will be rapidly scattered. Thus dispersion will take place instead of propagation.

Among N vertical obstacles of similar size but of different shape, subject to wave action, experience shows that a straight and rogular cylindrical structure should produce a minimm scattering of waves.

However, when an isolated structure is built at a fairly great depth, it is necessary to take into account the stability factor of the structure and its resistance to waves. Usually, a structure isolated at sea will be anchored on a mattress with dowels. Such a structure will then act as a vertical circular beam fixed at one extremity and subject to a system of forces, the resultant of which is horizontal. The kinetic energy developed by the force will then be absorbed by bending so that if $\mathrm{R}$ is the reaction to the force, one must have

$$
\frac{1}{2} m v^{2}=\frac{R}{2} \cdot a
$$

where $a=$ bending deflection

$$
\mathbf{x}=\text { mass of water of velocity } \mathbf{v} \text {. }
$$

The term a is a function of Ioung's modulus and of the beam length $\ell$. Although perhaps an ideal structure would consist of a cylinder of relatively small diameter with respect to the wave length, it will be realized that such a structure would offer in large depths rather precarious stability conditions because it would offer a small section modulus.

A pyramidal or conical shape, which allows the centre of gravity to be placed low enough, appears for this reason to be preferable. Onder these conditions, a well-anchored structure will resist wave action better Since a pyramidal structure offers more scattering than a cylindrical one of similar size, the wave run-up and resistance to shear due to drifting ice appear to remain the major problems to solve.

\section{MODEL STUDY}

The model study of a pier of conical shape to be used as the base of a lighthouse (at Prince Shoal, Quebec) was.carried out for the Department of Transport, Ottawa, to observe wave run-up conditions over the square 


\section{MODEL STUDY OF AN ISOLATED LIGHTHOUSE \\ PLATFORM AT SEA (PRINCE SHOAL, QUEBEC)}

platform (Fig. 1). The depth at low tide was $25 \mathrm{ft}$, the total height of the pier being $65 \mathrm{ft}$. The structure had been calculated to offer satisfactory safety coefficient against shear.

Waves were generated in a wave canal; the wave data available indicating a maximum observed wave hoight of $16 \mathrm{ft} .$, the periods varying between 5 and 11 seconds. At high tide ( $+15 \mathrm{ft}$. ), the tests showed that the amount of seattering observed for all periods was fairly high, the wave run-up caused by the folding front breaking at the structure, sometimes reaching $20 \mathrm{ft}$. above the square platform.

Opon completion of the test serles, it was decided that the proposed shape was inadequate on the basis of wave run-ap considerations. A diamond shape pier was an improvement, but was still not satisfactory.

The problem was then approached from the following viewpoint.

Since a frall cylindrical shape could not be retained, it was necessary to decrease the wave run-up to dissipate as mach as possible by some means the energy present in the wave run-up. To initiate this dissipation of energy, the principle of the flat slope could be retained provided that the structural qualities of the structure would not be impaired. The flat slope would create an instability of the wave front, thereby causing a partial collapse of the wave front before it impinged on the underside of the platform.

A final shape was amplved at (Fig. 2), and tests were run folloring the same procedure as for previous tests. It was observed (at high tide) that:

1. The pertrubation created by the presence of the flat slope increased the internal turbulence of the wave before it reached the cylindrical wall situated beneath the platform.

2. Part of the wave broke afterward at the cylindrical wall.

3. The remaining energy was such that an intense rortex circulation appeared around the cylinder and diffused into a wake in the shadow of the structure.

4. The energy reflected by the cylindrical wall was well checked by the curved part of the platform and a sheet of water was observed, which was perfectly reflected in the opposite direction to the incident wave.

A tentative explanation of the energy dissipation can be given as follows: At high tide, and for the high cambered waves, the partial or complete breading of the wave at or near the cylindrical wall creates an increase of the kinetic energy and an accelerative motion of the water. Since the cylindrical part is of a much smaller diameter than the base itself, it offers less scattering, allowing thereby relatively more energy to circulate around it than if the section were unchanged. There is, consequently, less energy per unit wave front reflected by the cylindrical base, and hence less energy available for the run-up. 


\section{COASTAL ENGINEERING}

The study of the motion around the obstacle, which is a solid of continuous curvature, is fairly complicated. For laminar conditions, the boundary layer starting from the stagnation point follows the surface for some distance, becomes turbulent, and then breaks away from the structure. For turbulent flow (in this case, this flow is also still partially oscillatory) it becomes more difficult to study the problem and only a check on the pressure distribution could yield some ideas about the circulation pattern obtained. It was also observed that the dispersion in the wake was very intense and rapid, but that the oscillatory character of the wave reappeared very quickly as the wave travelled away from the structure.

At low tide, the wave run-up appeared noticeably reduced and did not seem to constitute any problem. The scattering is then entirely controlled by the base of the structure while the upper part of the wave front is partially dissipated by turbulent shear flow induced by the breaking wave along the flat slope.

\section{REMARKS}

The possibliity of impulse pressures developing at the cylindrical wall, due to the breaking of the waves, does not constitute any danger since the peak pressures occur over a small area and for a very short time. They cannot seriously affect a monolithic structure and the possibility of resonance phenomena developing, due to elastic waves induced at the time of the breaking, appears remote in view of the large mass offered by the obstacle to the wave. This question would, horever, require a special study using a special model on which impulse pressures could be mechanically applied, the vibration being recorded with sensitive elements similar to strain-gauges.

Flat sides were adopted to allow ships to lie alongside the structure. These flat sides should be oriented in the direction of the highest waves. It is felt that under such conditions the probability of an important clapotis along these sides appears very remote since waves do not have the necessary fetches in which to grow. These sides provide facilities for shipping and will be useful in improving mooring condttions. It is realized that with a circulation inducing a current around a cireular structure, a ship which would be tangent to the structure at one point would tend to oscillate and slide axound it. These conditions are, hence, not favourable from the point of view of docking and mooring alongside.

\section{ICE CONDITIONS}

The cylindrical part of the structure below the platform was calculated to resist a shear force of 400 p.s.i. created by drifting ice. The calculation of resistance to shear was found sufficient. It is thought that the adopted slopes should facilitate the natural removal of the ice depositing at low tide. The buoyancy of the rising water would be sufficient to float the masses of ice and break them from the structure. Tidal currents would afterward cause the ice to drift away from the structure. 
MODEL STUDY OF AN ISOLATED LIGHTHOUSE

PLATFORM AT SEA (PRINCE SHOAL, QUEBEC)

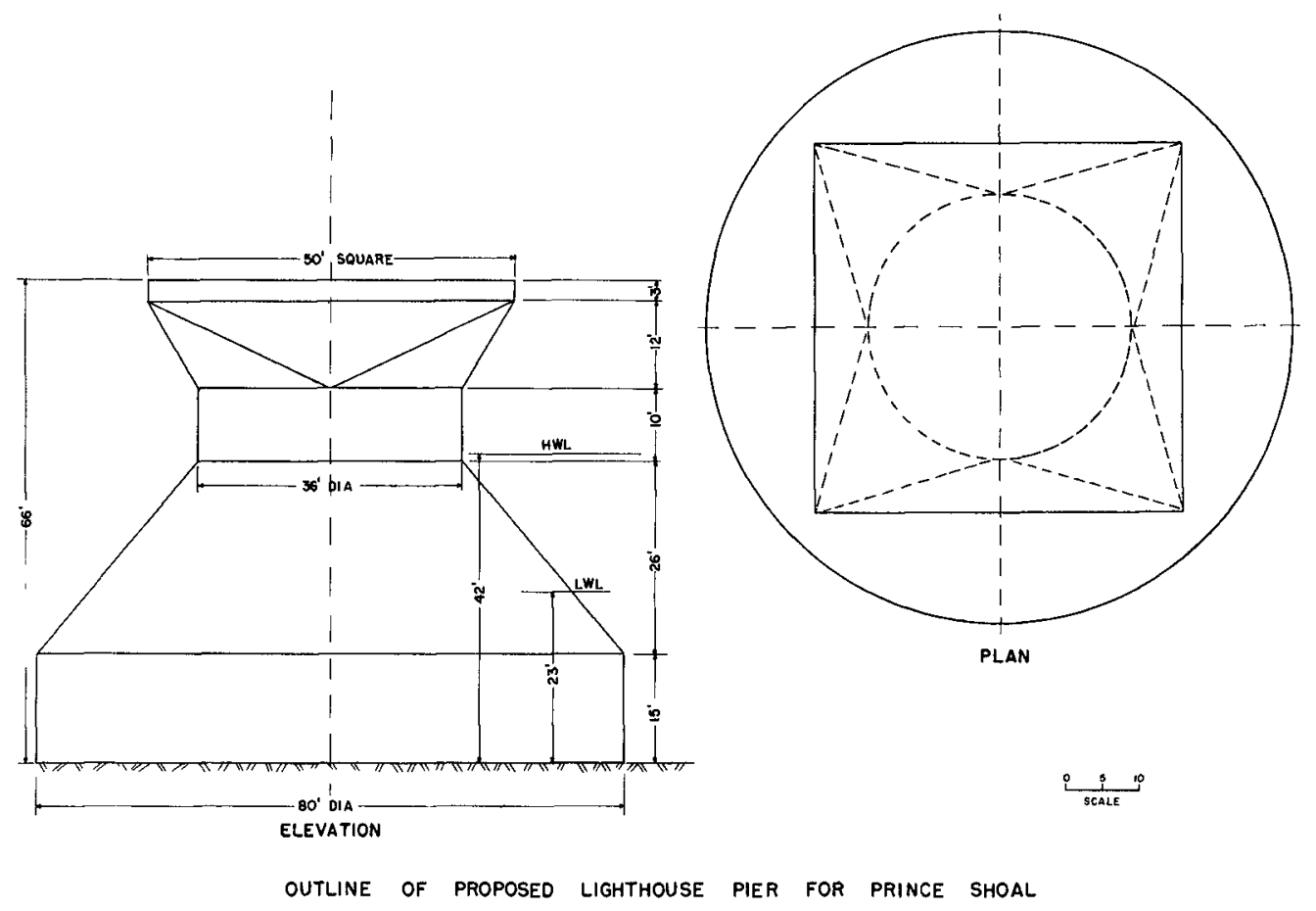

Fig. 1

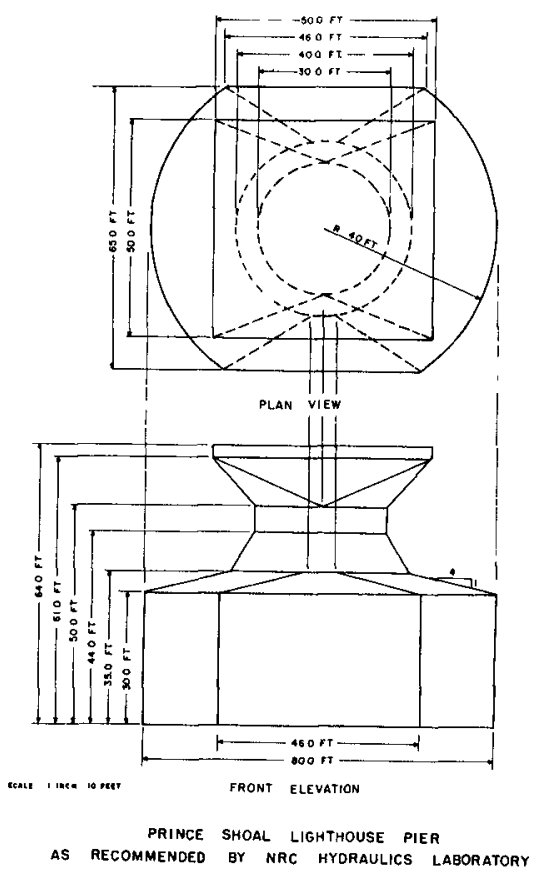

Fig. 2

783 


\section{COASTAL ENGINEERING}

\section{TOWING CONDITIONS OF THE BALLASTED SHELL}

The towing properties of the shell were studied in the towing tank of the Ship Iaboratory, on a model of $1 / 30$ scale. The lateral stability of the ressel was found excellent. A yewing motion was observed on either side of the centre line with a total amplitude never greater than $100 \mathrm{ft}$. $A$ ballast equivalent to about 24,00 tons was used yielding a comesponding draught of $24 \mathrm{ft}$. The best speed from the point of view of lateral stability and yaw was found to be 4.9 knots.

\section{BIBLIOGRAPHY}

1. Morison, J.R., O'Brien, M.P., Johnson, J.W. and Schaaf, S.A. "The Force Exerted by Surface Wares on Piles". J. Petroleum Technology, Vol. 2, No. 5, May 1950. (Trans.), p. 149 - 54.

2. Rayleigh, J.W.S., baron; "The Theory of. Sound". Dover, N.I. 2nd edit. 1945. 